\title{
Neutrino Oscillations v.s. Leptogenesis in SO(10) Models
}

\author{
Emmanuel Nezri \& Jean Orloff \\ Laboratoire de Physique Corpusculaire \\ F-63177 Aubière Cedex
}

\begin{abstract}
We study the link between neutrino oscillations and leptogenesis in the minimal framework assuming an $S O(10)$ see-saw mechanism with 3 families. Dirac neutrino masses being fixed, the solar and atmospheric data then generically induce a large mass-hierarchy and a small mixing between the lightest right-handed neutrinos, which fails to produce sufficient lepton asymmetry by 5 orders of magnitudes at least. This failure can be attenuated for a very specific value of the mixing $\sin ^{2} 2 \theta_{e 3}=0.1$, which interestingly lies at the boundary of the $\mathrm{CHOOZ}$ exclusion region, but will be accessible to future long baseline experiments.
\end{abstract}

KEywords: Baryogenesis, Solar and Atmospheric Neutrinos, Cosmology of

Theories beyond the SM. 


\section{Contents}

1. Introduction 1

2. Leptogenesis 3

3. $\mathrm{SO}(10)$

4. Toy see-saw (2 flavours) 9

5. Results with 3 flavours 13

6. Conclusions and perspectives 18

\section{Introduction}

The evidence for a total asymmetry between baryon and anti-baryon densities in our surroundings is an indisputable fact of life. This could easily be accounted for by assuming that the initial conditions for our universe are such that this asymmetry is of order one. However, there is every reason to believe that the thermal history of the universe can be traced back in time using known particle physics up to temperatures of maybe $100 \mathrm{GeV}$, certainly high enough to massively produce quark-antiquark pairs. At these temperatures, big-bang nucleosynthesis requires [1] that the adiabatic invariant ratio

$$
Y_{B} \doteq \frac{n_{B}-n_{\bar{B}}}{s}=0.710^{-10}
$$

meaning that there be about one extra quark for $10^{10}$ quark-antiquark pairs. Avoiding such extreme fine-tuning by a dynamical mechanism able to produce this number out of an initially symmetric configuration is the purpose of baryogenesis.

Many mechanisms of baryogenesis have been proposed in the past 30 years (see e.g. [2] for a review including the most ingenious and exotic ones), but most rely on ad-hoc new physics. We certainly would prefer to explain the baryon asymmetry from solid, experimentally tested physics. The standard model of particle physics satisfy this criterion at a desperately high level of precision, and it was shown [3] (see [4] for a review) to satisfy in principle all the Sakharov [5] conditions required to produce a baryon asymmetry. However, what looked like a very small number for initial conditions, now appears too large for the pure standard model to achieve. 
At least, $\mathrm{CP}$ violation beyond the known CKM phase must be added to resist the strong GIM cancellations in the hot plasma [6], [7].

Furthermore, the only possibility to change baryon number in the standard model is via sphaleron processes which should freeze abruptly below the electroweak phase transition to leave a net asymmetry [8]. Since the transition gets weaker when rising the mass of a single scalar doublet, extra non-standard scalars are invoked to counter the rising of the experimental lower bound on the lightest scalar. Supersymmetry naturally provides a lot of well-motivated extra scalars, but getting a strong enough transition with $m_{H} \approx 100 \mathrm{GeV}$ requires a huge gap between the right-handed stop and all other sfermions, which may seem contrived, and even so, could not protect E-W baryogenesis against a $\approx 110 \mathrm{GeV}$ Higgs bound [9]. Unless a scalar is soon discovered, we thus seem to be lead back into the pre-[10] situation, where baryogenesis was a footprint from extreme high-energy physics, with little chance of an experimental cross-check.

However, another experimental signal for non-standard physics has since then developed into a quantitative and solid field, namely the evidence for neutrino oscillations which strongly points toward small but non-zero neutrino masses [1]. If these masses are of the Dirac type, right-handed neutrinos must be added, but we are left with the puzzle: why are neutrinos $10^{10}$ times lighter than charged leptons? In our mind, the smallest theoretical price to pay for resolving this puzzle is to keep the right-handed neutrinos, but give them large Majorana masses $\approx 10^{10-16} \mathrm{GeV}$ : this is the celebrated see-saw mechanism [12], [13], [14, [15]. Such a high Majorana mass breaks lepton number at a slow enough rate, and in a very indirect way, one can say that neutrino oscillations provide a leptonic analogue of the baryon number violation looked for unsuccessfully in proton decay searches during the last decades.

With this theoretical prejudice, we are thus lead to take the existence of heavy right-handed neutrinos for granted. This makes leptogenesis [16] an extremely natural mechanism to consider for producing the baryon asymmetry. Indeed, the decay of right-handed neutrinos can easily leave a CP-odd lepton asymmetry, which standard sphaleron processes can convert into a baryon asymmetry. It is then most interesting to see how closely can this asymmetry be related to tested or testable neutrino oscillation physics, and how much asymmetry can be produced.

To study these questions, we first review in section 2 the generic leptogenesis mechanism, to fix notations and give the formula for the asymmetry in terms of neutrino masses. Then we focus in section 5 on a generic class of 3 families $S O(10)$ models offering a computable connection between leptogenesis and neutrino oscillations. In section 4 , we analytically work out this connection in a toy see-saw model with only 2 families, to help and understand the results presented in section 5 . 


\section{Leptogenesis}

One of the most attractive scenario to explain the baryon asymmetry of the universe is leptogenesis. In such a scenario, a primordial lepton asymmetry is generated by the out-of-equilibrium decay of heavy right-handed Majorana neutrinos $N_{i}$. The possible excess in lepton number $L$ is converted into a baryon asymmetry $B$ by the sphaleron processes. From relations between chemical potentials this conversion is given by (see [17, 18] and references therein):

$$
B=\frac{8 N_{F}+4 N_{H}}{22 N_{F}+13 N_{H}}(B-L)
$$

where $N_{F}$ and $N_{H}$ are respectively the number of families and Higgs doublets.

At low energies, right-handed neutrinos $\left(N_{i}\right)$ are gauge-singlets, and only appear in the Lagrangian via their Majorana masses $\left(M_{i}\right)$ and Dirac Yukawa couplings $\left(h_{l i}\right)$ to the Higgs doublet $(H)$ and lepton doublet $\left(L_{l}\right)$

$$
\mathcal{L}=\mathcal{L}_{M}+\mathcal{L}_{Y}=\frac{1}{2} \overline{\left(N_{i}\right)^{c}} M_{i} N_{i}+\overline{L_{l}} i \sigma_{2} H^{*} h_{l i} N_{i}+h . c
$$

in the right-handed neutrinos eigenbasis $\left(0<M_{1} \leq M_{2} \leq M_{3}\right)$. By the see-saw mechanism, light neutrinos then get a Majorana mass-matrix :

$$
m_{\nu}=v^{2} h \cdot \operatorname{Diag}\left(\frac{1}{M_{1}}, \frac{1}{M_{2}}, \frac{1}{M_{3}}\right) \cdot h^{T}
$$

The 3 Sakharov conditions are easily met by the $N$-decays. Indeed, the right Majorana mass fulfils the first condition by violating lepton number $L$, manifest in the simultaneous existence of both decays channels $N_{i} \rightarrow H+l$ and $N_{i} \rightarrow H^{\dagger}+\bar{l}$.

Second, the CP violation condition can be met by the interference between treelevel and $\epsilon^{\prime}$ (vertex) or $\epsilon$ (wave function) one-loop corrections. Indeed, CP asymmetry in the decay (in vacuum) of the right-handed neutrino $N_{i}$ is [19, 20]:

$$
\delta_{i}=\frac{\sum_{l} \Gamma\left(N_{i} \rightarrow l+H\right)-\Gamma\left(N_{i} \rightarrow \bar{l}+H^{\dagger}\right)}{\sum_{l} \Gamma\left(N_{i} \rightarrow l+H\right)+\Gamma\left(N_{i} \rightarrow \bar{l}+H^{\dagger}\right)}=\sum_{j} \epsilon_{i, j}+\epsilon_{i, j}^{\prime}
$$

with

$$
\epsilon_{i, j}=-\frac{1}{8 \pi} \frac{\operatorname{Im}\left(\sum_{l} h_{l i}^{*} h_{l j}\right)^{2}}{\sum_{l}\left|h_{l i}\right|^{2}} \frac{M_{i} M_{j}}{M_{j}^{2}-M_{i}^{2}} ; \epsilon_{i, j}^{\prime}=\frac{1}{8 \pi} \frac{\operatorname{Im}\left(\sum_{l} h_{l i}^{*} h_{l j}\right)^{2}}{\sum_{l}\left|h_{l i}\right|^{2}} f\left(\frac{M_{j}^{2}}{M_{i}^{2}}\right)
$$

where $f(x)=\sqrt{x}\left[1-(1+x) \ln \left(\frac{1+x}{x}\right)\right]$. Defining

$$
A_{i j}=\left(h^{\dagger} h\right)_{i j}
$$


the virtual $N_{j}$ contribution to the $N_{i}$ decay asymmetry becomes for a large hierarchy ${ }^{1}$ between heavy Majorana neutrinos $M_{i} \ll M_{j}$

$$
\epsilon_{i, j}=-\frac{1}{8 \pi} \frac{\operatorname{Im}\left(A_{i j}^{2}\right)}{A_{i i}} \frac{M_{i}}{M_{j}} ; \epsilon_{i, j}^{\prime}=-\frac{1}{16 \pi} \frac{\operatorname{Im}\left(A_{i j}^{2}\right)}{A_{i i}} \frac{M_{i}}{M_{j}}
$$

while in the opposite limit $M_{i} \gg M_{j}$ :

$$
\epsilon_{i, j}=-\frac{1}{8 \pi} \frac{\operatorname{Im}\left(A_{j i}^{2}\right)}{A_{i i}} \frac{M_{j}}{M_{i}} ; \epsilon_{i, j}^{\prime}=\frac{1}{4 \pi} \frac{\operatorname{Im}\left(A_{j i}^{2}\right)}{A_{i i}} \frac{M_{j}}{M_{i}} \ln \left(\frac{M_{j}}{M_{i}}\right) .
$$

Concerning the third Sakharov condition, let us first assume $N_{i}$ to be thermally distributed in the early universe when $T \geq M_{i}$. While cooling, the equilibrium distribution starts feeling a strong Boltzmann suppression when $T \approx M_{i}$. If the vacuum decay rate $\Gamma_{N_{i}}=\frac{1}{8 \pi} A_{i i} M_{i}$ was much slower than the expansion rate at that time $H\left(M_{i}\right)=1.66 \sqrt{g^{*}} \frac{M_{i}^{2}}{M_{p l}}$, each $N_{i}$ would decay essentially in vacuum and starting from $\frac{n_{i}}{n_{\gamma}} \approx 1$, we would get [23] $Y_{i}=L_{i} / s \approx \delta_{i} / g^{*}$. However, decay rates are not necessary that slow, and if the universe is still hot by the time $N_{i}$ decays, inverse decays dilute $\delta_{i}$ roughly into $\delta_{i} / K_{i}$ with :

$$
K_{i}=\frac{\Gamma_{N_{i}}\left(T=M_{i}\right)}{H\left(T=M_{i}\right)} \approx \frac{1}{8 \pi 1.66 \sqrt{g^{*}}} A_{i i} \frac{M_{p l}}{M_{i}}
$$

characterising the efficiency of (inverse-)decay processes when $N_{i}$ becomes non-relativistic. It is convenient 24] to rewrite this dimensionless parameter in terms of an effective see-saw like mass

$$
\tilde{m}_{i} \doteq \frac{v^{2} A_{i i}}{M_{i}}=K_{i} \tilde{m}^{*}
$$

with a critical value $\tilde{m}^{*}=\sqrt{512 g^{*} \pi^{5} / 90} v^{2} / M_{p l}=1.0810^{-3} \mathrm{eV}$ in the Standard Model. Below this critical value, decays happen like in vacuum, but the Yukawa couplings are too small for the $N_{i}$ population to reach equilibrium, and the result depends on the choice of initial conditions. If we assume no initial population, there is also a suppression for $\tilde{m}_{i}<\tilde{m}^{*}$, and the final asymmetry

$$
Y_{i}=\frac{\delta_{i}}{g^{*}} d\left(\tilde{m}_{i}, M_{i}\right)
$$

contains a dilution factor $d(\tilde{m})$ that is only close to 1 for $\tilde{m} \approx \tilde{m}^{*}$. For $M_{i} \leq 10^{8} \mathrm{GeV}$ where 2 body scatterings can be neglected, the solution of the Boltzmann equations (fig. 6 of [25]) can be reasonably fitted by

$$
d(\tilde{m})=\frac{1}{\left(\tilde{m} / 10 \tilde{m}^{*}\right)^{-0.8}+\left(\tilde{m} / 0.28 \tilde{m}^{*}\right)^{1.25}}
$$

which is compared with the usual approximation $23 d(K>10) \approx 0.3\left(K \ln ^{0.6} K\right)^{-1}$ on figure 1] (see also [26]).

${ }^{1}$ The expressions (2.5) anyway require resumming in the degenerate limit, as the asymmetry should then vanish instead of diverging 21, 20, 18, 22]. 


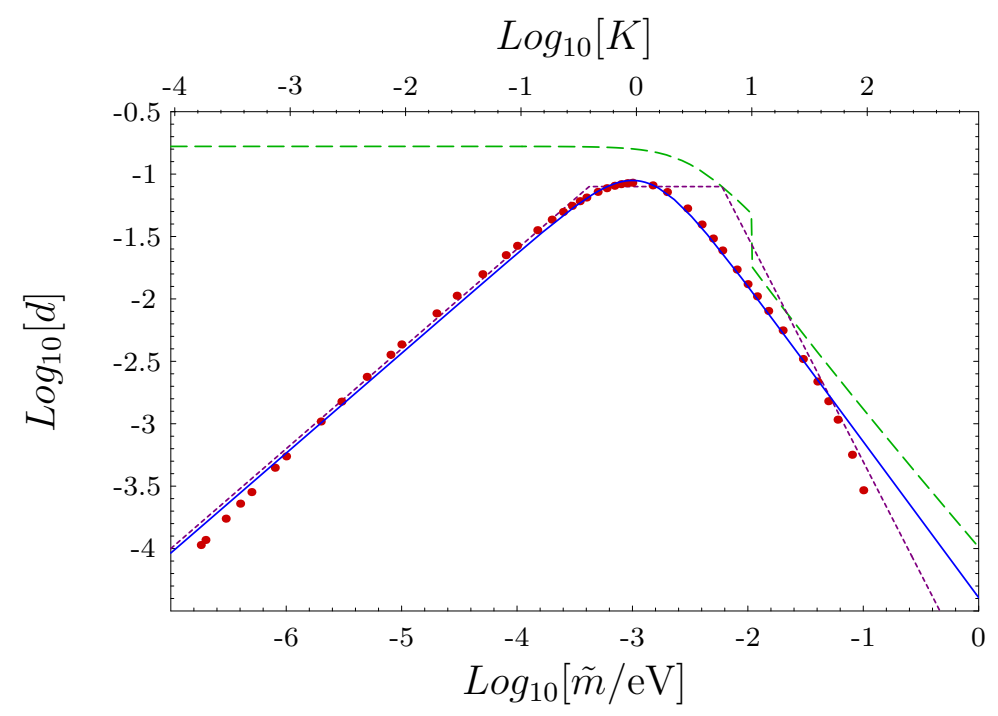

Figure 1: Fits of the dilution factor $d$ computed by 25] in the SM for $M_{1}=10^{8} \mathrm{GeV}$ (red dots): the plain blue curve is our formula (2), to be compared with: 1) the usual approxmation $0.3\left(K \ln ^{0.6} K\right)^{-1}$ for large $K$ from [23], extended to lower $K$ 's following [27] (green dashed curve); 2) the expression of [28], more appropriate for larger $M_{1}$ (purple dotted curve).

Decay of the lightest $N_{1}$ usually gives the latest and dominant contribution, because comparing (2.7) and (2.8) shows that other $N_{j}$ 's are suppressed by $A_{11}^{2} / A_{j j}^{2}$ (typically $10^{-10}$ ) and further diluted by scattering processes (see for instance [29] for a discussion of such processes). Using $Y_{B} \approx-1 / 3 Y_{L}$ from sphaleron conversion (2.1), the final baryon asymmetry we use in section 5 is then:

$$
Y_{B 10} \doteq 10^{10} Y_{B} \approx-\frac{10^{10}}{3 g^{\star}} d\left(\tilde{m}_{1}, M_{1}\right) \frac{-3 M_{1}}{16 \pi A_{11}} \sum_{j=2,3} \frac{\operatorname{Im}\left(A_{1 j}^{2}\right)}{M_{j}} \approx 0.7
$$

At this level, no constraints from neutrino oscillations encoded in (2.3) can be drawn because the Yukawa coefficients $h$ are free parameters: for any set of parameters $(h, M)$ providing good oscillation physics but bad $Y_{B 10}$, we can rescale $h \rightarrow h^{\prime}=h / \sqrt{Y_{B 10}}$ and $M \rightarrow M^{\prime}=M / Y_{B 10}$ to get the same light neutrinos, with $Y_{B 10} \approx 1$. Notice that $K$ and $\tilde{m}$ are invariant under such rescaling, so that the only violation to this scaling law comes from the $M_{1}$ dependence of the dilution factor $d$.

To set the $M_{1}$ scale of interest, it is instructive to make the further approximation $d\left(1<K<10^{3}\right) \approx 0.1 / K$ and assume domination of the $N_{1}$ decay with a virtual $N_{2}$ exchange. The result for the asymmetry is then

$$
Y_{B 10} \approx \frac{0.7 \times 10^{-10}}{\sqrt{g^{*}}} \frac{\operatorname{Im}\left(A_{12}^{2}\right)}{A_{11}^{2}} \cdot \frac{M_{1}}{M_{2}} \cdot \frac{M_{1}}{10^{10} \mathrm{GeV}} \approx 0.7
$$

so that $M_{1} \approx 10^{10} \mathrm{GeV}$ is the natural scale for leptogenesis. 
To draw any link between neutrino oscillations and leptogenesis, we thus need further theoretical assumptions, of which we now describe our minimal set.

\section{3. $\mathrm{SO}(10)$}

$S O(10) \supset S U(3) \times S U(2) \times U(1)$

The $S O(10)$ Grand Unification group is a very natural framework for studying leptogenesis. Indeed [30, 31], it is the smallest GUT group including right-handed neutrinos together with usual fermions in matter multiplets :

$$
\underbrace{16}_{\psi}=\underbrace{(3,2)}_{Q}+\underbrace{(\overline{3}, 1)}_{u_{R}^{c}}+\underbrace{(\overline{3}, 1)}_{d_{R}^{c}}+\underbrace{(1,2)}_{L}+\underbrace{(1,1)}_{e_{R}^{c}}+\underbrace{(1,1)}_{N}
$$

Furthermore, it includes $(B-L)$ as a gauge symmetry, whose breaking gives a natural explanation for right-handed neutrino Majorana masses. Finally, leptons and quarks masses are related which fixes the neutrino Dirac Yukawa $h$. For instance, if Dirac masses originate from a single scalar in the vector (10-dimensional) of $S O(10)$ :

$$
\mathcal{L}_{Y}=f_{a b}^{10} \psi_{a}^{T} \Gamma_{A} \psi_{b} \phi_{A}^{10}
$$

we obtain the (unphysical) low energy relation :

$$
m_{d}=3 m_{e}=m_{u}=3 m_{\nu} \propto f^{10} v^{10}
$$

where the factor of about 3 comes from the extra color loops affecting quarks but not leptons. Adding a second (or more) scalars in the same $S O(10)$ representation :

$$
\mathcal{L}_{Y}^{\prime}=f_{a b}^{\prime 10} \psi_{a}^{T} \Gamma_{A} \psi_{b} \phi_{A}^{\prime 10}
$$

lifts the degeneracy $m_{u}=m_{d}$ while keeping $m_{u} \approx 3 m_{\nu}$ i.e $m_{\nu} \approx 1 \mathrm{MeV} \rightarrow 50 \mathrm{GeV}$.

To get more realistic neutrino masses, an extra scalar $\phi^{126}$ in the 126-representation with v.e.v $v_{126}$ in the $S U(5)$-singlet component can be invoked to break $(B-L)$, providing the Majorana mass term :

$$
\mathcal{L}_{M}=f_{a b}^{126} v_{126} \overline{N_{a}^{c}} N_{b}+\text { h.c }
$$

Thus, in the framework of $S O(10)$, Dirac masses are naturally determined by upquarks masses.

\section{$A_{i j}$ expression}

Writing the relevant Lagrangian term for the leptons we get after $S U(2)$ breaking :

$$
\mathcal{L}=\left[\overline{L_{a}} \times\left(\begin{array}{c}
0 \\
v^{d}
\end{array}\right)\right] f_{a b}^{d} l_{R b}+\left[\overline{L_{a}} \times\left(\begin{array}{c}
v^{u} \\
0
\end{array}\right)\right] f_{a b}^{u} N_{b}+\frac{1}{2} \overline{N_{a}^{c}} v_{126} f_{a b}^{126} N_{b}+h . c
$$


so that $M_{a b} \doteq f_{a b}^{126} v_{126}$ with $a$ and $b$ being components in some arbitrary family basis. Decomposing the Yukawa couplings into mixing and real masses eigenvalues, we write :

$$
\begin{aligned}
f^{d} & =\frac{1}{3 v^{d}} U_{L d}^{\dagger} \cdot \operatorname{Diag}\left(m_{d}, m_{s}, m_{b}\right) \cdot U_{R d} \\
f^{u} & =\frac{1}{3 v^{u}} U_{L u}^{\dagger} \cdot \operatorname{Diag}\left(m_{u}, m_{c}, m_{t}\right) \cdot U_{R u} \\
f^{126} & =\frac{1}{v^{126}} U_{M}^{*} \cdot \operatorname{Diag}\left(M_{1}<M_{2}<M_{3}\right) \cdot U_{M}^{\dagger}
\end{aligned}
$$

The previous $h_{l i}$ are up-Yukawa matrix elements between charged leptons mass (and flavour) eigenstates $\left(l_{l}\right)$ and right-handed neutrinos mass eigenstates $\left(N_{i}\right)$. Therefore:

$$
\begin{aligned}
h_{l i} & =U_{L d} \cdot f^{u} \cdot U_{M} \\
& =\frac{1}{3 v^{u}} U_{L d} \cdot U_{L u}^{\dagger} \cdot \operatorname{Diag}\left(m_{u}, m_{c}, m_{t}\right) \cdot U_{R u} \cdot U_{M} \\
& \doteq \frac{1}{3 v^{u}} V_{C K M}^{\dagger} \cdot \operatorname{Diag}\left(m_{u}, m_{c}, m_{t}\right) \cdot V_{R}
\end{aligned}
$$

so that

$$
A_{i j}=\frac{1}{9\left(v^{u}\right)^{2}} V_{R}^{\dagger} \cdot \operatorname{Diag}\left(m_{u}^{2}, m_{c}^{2}, m_{t}^{2}\right) \cdot V_{R}
$$

only depend on the unknown $V_{R}$ mixing. But the light neutrinos mass matrix is constrained by neutrinos oscillation physics:

$$
\begin{aligned}
m_{\nu} & =v_{u}^{2} h \cdot \operatorname{Diag}\left(\frac{1}{M_{1}}, \frac{1}{M_{2}}, \frac{1}{M_{3}}\right) \cdot h^{T} \\
& =U \cdot \operatorname{Diag}\left(m_{1}, m_{2}, m_{3}\right) \cdot U^{T}
\end{aligned}
$$

The unknowns $\left(V_{R}, M_{1,2,3}\right)$ can thus be determined from measurable quantities $\left(U, m_{1,2,3}\right)$ by diagonalizing the matrix :

$$
\begin{aligned}
M_{R}^{*} & =\frac{1}{9} \operatorname{Diag}\left(m_{u}, m_{c}, m_{t}\right) \cdot U_{e f f} \cdot \operatorname{Diag}\left(\frac{1}{m_{1}}, \frac{1}{m_{2}}, \frac{1}{m_{3}}\right) \cdot U_{e f f}^{T} \cdot \operatorname{Diag}\left(m_{u}, m_{c}, m_{t}\right)(3 \cdot 1 \\
& =V_{R} \cdot \operatorname{Diag}\left(M_{1}, M_{2}, M_{3}\right) \cdot V_{R}^{T}
\end{aligned}
$$

where

$$
U_{e f f} \doteq V^{C K M} \cdot U
$$

\section{Parameterisation \& experimental inputs:}

Using the standard parameterisation 32 for $V^{C K M}$ :

$$
V^{C K M}=V_{23} \cdot V_{13} \cdot V_{12}=\left(\begin{array}{ccc}
c_{12} c_{13} & s_{12} c_{13} & s_{13}^{*} \\
-s_{12} c_{23}-c_{12} s_{23} s_{13} & c_{12} c_{23}-s_{12} s_{23} s_{13} & s_{23} c_{13} \\
s_{12} s_{23}-c_{12} c_{23} s_{13} & -c_{12} s_{23}-s_{12} c_{23} s_{13} & c_{23} c_{13}
\end{array}\right)
$$


we use the following values for the quark mixing angles:

$$
\begin{aligned}
s_{23}^{C K M} & =0.041 \\
s_{13}^{C K M} & =0.0036 e^{i \delta^{C K M}} ; \delta^{C K M} \approx 1 \\
s_{12}^{C K M} & =0.223
\end{aligned}
$$

As for light neutrinos 33, we label mass eigenstates so that the most constraining experiments each fix a definite mixing angle and use a similar decomposition with three complex mixing angles, and Majorana phases:

$$
\begin{aligned}
U & =\underbrace{U_{23}}_{\text {atm }} \cdot \underbrace{U_{13}}_{\text {Chooz }} \cdot \underbrace{U_{12}}_{\text {sun }} \cdot \operatorname{Diag}\left(1, e^{i \phi_{1}}, e^{i \phi_{2}}\right) \\
& =\left(\begin{array}{ccc}
c_{\text {sun }} c_{e 3} & s_{12}^{*} c_{e 3} & s_{13}^{*} \\
-s_{12} c_{a t m}-c_{\text {sun }} s_{23}^{*} s_{13} & c_{\text {sun }} c_{a t m}-s_{12}^{*} s_{23}^{*} s_{13} & s_{23}^{*} c_{e 3} \\
s_{12} s_{23}-c_{\text {sun }} c_{\text {atm }} s_{13} & -c_{\text {sun }} s_{23}-s_{12}^{*} c_{a t m} s_{13} & c_{a t m} c_{e 3}
\end{array}\right) \cdot\left(\begin{array}{ccc}
1 & 0 & 0 \\
0 & e^{i \phi_{1}} & 0 \\
0 & 0 & e^{i \phi_{2}}
\end{array}\right)
\end{aligned}
$$

where the complex parameters are:

$$
\begin{aligned}
& s_{12}=s_{\text {sun }} e^{i \delta_{12}} \\
& s_{13}=s_{e 3} e^{i \delta_{13}} \\
& s_{23}=s_{\text {atm }} e^{i \delta_{23}}
\end{aligned}
$$

Squared mass differences are then fixed (up to a sign) by the oscillation parameters $\Delta m_{\text {sun }}^{2} \ll \Delta m_{\text {atm }}^{2}$ :

$$
\begin{aligned}
& m_{2}^{2}-m_{1}^{2}=\Delta m_{\text {sun }}^{2} \\
& m_{3}^{2}-m_{1}^{2} \approx m_{3}^{2}-m_{2}^{2}= \pm \Delta m_{a t m}^{2}
\end{aligned}
$$

In both cases, $m_{3}$ is singled out by atmospheric oscillations, but according to the sign, it can either be heavier

$$
m_{1} \leq m_{2} \ll m_{3} \quad \text { (standard hierarchy), }
$$

or lighter than the others

$$
m_{3} \ll m_{1} \leq m_{2} \quad \text { (inverted hierarchy). }
$$

We take the lightest mass $\left(m_{1}\right.$ or $\left.m_{3}\right)$ as the free parameter that cannot be fixed by oscillations. For values of this parameter larger than $\Delta m_{a t m}$, we get two different realizations of the degenerate case. In the following, it will vary between $10^{-7} \mathrm{eV}$ and $1 \mathrm{eV}$ : going below this range requires huge right-handed masses, typically above GUT or even Planck scale; going above does not help to increase the asymmetry, and 
generally violates neutrino-less double beta decay experiments 34, 35] (see however [36] for a study of possible cancellations using Majorana phases).

Other free parameters are the six phases: $\delta^{C K M}$ (from $V^{C K M}$ ), $\delta_{12}, \delta_{13}, \delta_{23}$ (from the complex mixing angles ${ }^{2}$ in $U$ ), and the two Majorana phases $\phi_{1,2}$. A careful count of the 6 phases in see-saw models can be found in reference [37].

With the above parametrisation, the experimental constraints from oscillations lead us to take the following values in the subsequent numerical analysis:

- Atmospheric neutrinos 32]: $\Delta m_{\text {atm }}^{2} \approx 310^{-3} \mathrm{eV}^{2}$ with nearly maximal mixing: $\sin ^{2} 2 \theta_{\text {atm }}=0.92 \rightarrow 1$ so that $s_{\text {atm }}=0.6 \rightarrow 0.7$.

- CHOOZ reactor experiment [38]: for the above value of $\Delta m_{a t m}^{2},\left|U_{e 3}\right| \doteq s_{e 3}<$ 0.18 at $95 \%$ C.L.

- Solar neutrinos [39]: we will consider 4 possible resolutions of the solar neutrino deficit:

1. large mixing angle MSW oscillations (LMA) with $\Delta m_{\text {sun }}^{2} \approx 510^{-5} \mathrm{eV}^{2}$ and $\tan ^{2} \theta_{\text {sun }}=0.42 \in[0.15,0.9]$ at $95 \%$ C.L. corresponding to $s_{\text {sun }}=0.54 \in$ $[0.36,0.69]$;

2. small mixing angle MSW oscillations (SMA) with $\Delta m_{\text {sun }}^{2} \approx 510^{-6} \mathrm{eV}^{2}$ and $\tan ^{2} \theta_{\text {sun }}=1.510^{-3} \in\left[610^{-4}, 310^{-3}\right]$ at $95 \%$ C.L. corresponding to $s_{\text {sun }}=0.039 \in[0.025,0.055]$;

3. LOW with $\Delta m_{\text {sun }}^{2} \approx 7.910^{-8} \mathrm{eV}^{2}$ and $\tan ^{2} \theta_{\text {sun }}=0.61$ corresponding to $s_{\text {sun }}=0.62$

4. vacuum oscillations (VAC) with $\Delta m_{\text {sun }}^{2} \approx 4.610^{-10} \mathrm{eV}^{2}$ and $\tan ^{2} \theta_{\text {sun }}=$ 1.8 so that $s_{\text {sun }}=0.8$;

Although the LMA solution is favoured by the SNO data 40] and confirmed by the latest KamLAND results [41], we still consider the SMA, LOW and VAC situations for completeness and illustration.

\section{Toy see-saw (2 flavours)}

Before reviewing the results in a full case with 3 families, it helps intuition to work out a 2 families exercise. In particular, equation (2.9) suggests that leptogenesis prefers right handed neutrinos with large mixing and similar masses. This is not easy to achieve by see-saw with large Dirac hierarchies and mixing 42, 43. Starting from the see-saw formula (3.14), dropping the first family and introducing the Dirac

\footnotetext{
${ }^{2}$ Notice that we take $\delta_{12,13,23} \in[-\pi / 2, \pi / 2]$, so that $s_{\text {sun }, e 3, \text { atm }} \in[-1,1]$ and $c_{\text {sun,e3,atm }} \in[0,1]$ instead of being restricted to the first quadrant like the usual CKM angles
} 
hierarchy parameter $r \doteq m_{c} / m_{t} \approx 10^{-2}$ for instance and the light neutrinos hierarchy parameter $m \doteq m_{2} / m_{3}$, we get in the case of real effective mixing parametrised by $s \doteq \sin \theta_{23}^{e f f}$

$$
\begin{aligned}
M_{R} & =\frac{1}{9}\left(\begin{array}{cc}
m_{c} & 0 \\
0 & m_{t}
\end{array}\right) \cdot\left(\begin{array}{cc}
c & s \\
-s & c
\end{array}\right) \cdot\left(\begin{array}{cc}
\frac{1}{m_{2}} & 0 \\
0 & \frac{1}{m_{3}}
\end{array}\right) \cdot\left(\begin{array}{cc}
c & -s \\
s & c
\end{array}\right) \cdot\left(\begin{array}{cc}
m_{c} & 0 \\
0 & m_{t}
\end{array}\right) \\
& =\frac{m_{t}^{2}}{9 m_{3}} \cdot\left(\begin{array}{cc}
r^{2}\left(c^{2}+s^{2} m\right) & r c s(m-1) \\
r c s(m-1) & c^{2} m+s^{2}
\end{array}\right) \cdot \frac{1}{m}
\end{aligned}
$$

For a large inverse hierarchy (w.r.t. Dirac hierarchy at small angle) $m \gg 1$, we get for small $r, s \ll 1$ (in units of $m_{t}^{2} / 9 m_{3}$ )

$$
M_{R} \approx\left(\begin{array}{cc}
r^{2} s^{2}+r^{2} / m & r s \\
r s & 1
\end{array}\right)=\left(\begin{array}{cc}
c_{R} & -s_{R} \\
s_{R} & c_{R}
\end{array}\right) \cdot\left(\begin{array}{cc}
M_{1} & 0 \\
0 & M_{2}
\end{array}\right) \cdot\left(\begin{array}{cc}
c_{R} & s_{R} \\
-s_{R} & c_{R}
\end{array}\right)
$$

so that $M_{2} \approx \operatorname{Tr} M_{R} \approx 1, M_{1} \approx \operatorname{Det} M_{R}=r^{2} / m \ll \operatorname{Tr} M_{R}$ while the right-handed mixing $s_{R}^{2} \approx r^{2} s^{2}$ is strongly suppressed by the Dirac hierarchy $r \ll 1$. The ratio appearing in the leptogenesis (2.9) is

$$
\frac{A_{12}}{A_{11}}=-\frac{c_{R} s_{R}}{r^{2} c_{R}^{2}+s_{R}^{2}} \approx-\frac{s}{r}
$$

and the the asymmetry $(2.10)$ goes in this case like

$$
Y_{B 10}^{a p p r} \propto \frac{A_{12}^{2}}{A_{11}^{2}} \cdot \frac{M_{1}}{M_{2}} \cdot M_{1} \approx \frac{r^{2} s^{2}}{m^{2}}
$$

which is strongly suppressed and dominated by the smallest values of $m$ possible. But in this degenerate region $m \approx 1$, another type of suppression arises as $s_{R}$ vanishes together with the off-diagonal terms in (4.2).

To hope for larger results, we must thus turn to the usual hierarchy limit $m \ll 1$. Assuming again $r, s \ll 1, M_{R}$ in (4.2) then becomes

$$
\left(\begin{array}{cc}
r^{2} & -r s \\
-r s & s^{2}+m
\end{array}\right) \cdot \frac{1}{m}
$$

By furthermore assuming $M_{1} \ll M_{2}$ (i.e. $r^{2} m \ll\left(m+r^{2}+s^{2}\right)^{2}$ which turns out to be satisfied everywhere, except when $s^{2} \ll r^{2} \approx m$ (see figure 2), we arrive at the useful analytic expressions

$$
\begin{aligned}
M_{2} & \approx \operatorname{Tr} M_{R} \approx \frac{1}{m}\left(m+r^{2}+s^{2}\right) \\
M_{1} & \approx \frac{\operatorname{Det} M_{R}}{\operatorname{Tr} M_{R}} \approx \frac{r^{2}}{m+r^{2}+s^{2}} \\
-A_{21} & =s_{R} c_{R} \approx \frac{-r s}{m+r^{2}+s^{2}}
\end{aligned}
$$




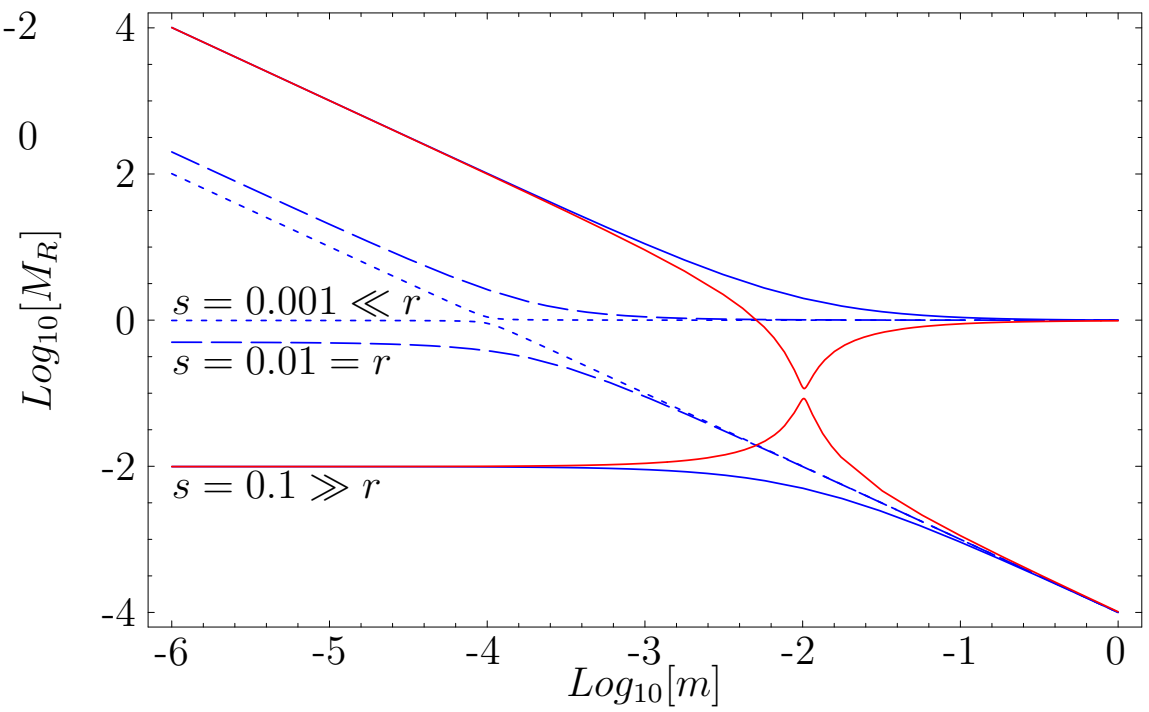

Figure 2: Right-handed masses $M_{R}$ in arbitrary units, as a functions of light neutrino masses ratio $m=m_{2} / m_{3}$, for 3 values of light neutrino effective mixing $s=\sin \left(\theta_{23 e f f}\right)=$ $10^{-1}, 10^{-2}, 10^{-3}$ and a fixed value of the Dirac masses hierarchy $r=m_{c} / m_{t}=0.01$. For $s=0.1$, we also show the effect of the Majorana phase $\phi_{m}=0.99 \pi$ (red curve) leading to degenerate Majorana masses.

From these expressions, it is clear that a cross-over occurs at values of $m$ around the largest of $s^{2}$ or $r^{2}$, but unlike the level-repulsion effect usual in quantum mechanics, the "levels" $M_{1,2}$ do not need to be close for the cross-over to happen, as can be seen in the $s>r$ situation. In this case, the levels do not asymptotically tend to the no-mixing situation away from cross-over region. These results are most simply summarised in the plots 2, drawn without approximation for $r=10^{-2}$.

Since $A_{11}=r^{2} c_{R}^{2}+s_{R}^{2}$, the ratio $A_{12} / A_{11}$ only takes a simple form for small mixing $s_{R} \ll r$ (large enough $m$ or small enough $s$ ), in which case the expression coming into the baryon asymmetry is simply

$$
Y_{B 10}^{a p p r} \propto \frac{A_{12}^{2}}{A_{11}^{2}} \cdot \frac{M_{1}}{M_{2}} \cdot M_{1} \approx \frac{m s^{2} r^{2}}{\left(m+r^{2}+s^{2}\right)^{5}},
$$

growing like $m \propto M_{2}^{-1}$ for small $m$, reaching a maximum around $r^{2}+s^{2}$ and decreasing like $m^{-4}$ beyond. We see in figure 3 that these approximations capture the general shape of the asymmetry, except for extra suppressions in the regions $m \approx 1$ (left-handed degenerate), and $m<s$ if $s^{2} \ll r^{2}\left(A_{11} \gg r^{2}\right)$, where the approximations used break down.

Of course, the true asymmetry $Y_{B 10}$ vanishes unless $A_{12}$ gets a CP-violating non-zero phase. Specialising (3.18) for 2 flavours leaves 2 phases, $\phi_{m} \doteq 2\left(\phi_{2}-\phi_{1}\right)$ and $\delta_{23}$, which respectively complexify the parameters $m=|m| e^{i \phi_{m}}, s=|s| e^{i \delta_{23}}$ and $s_{R}=\left|s_{R}\right| e^{i \delta_{R}}$ in the previous discussion. It is a bit tedious to check that the above approximations (4.4 4.6) for $M_{1} \ll M_{2}$ and $\left|A_{12}\right|$ can be extended to complex 


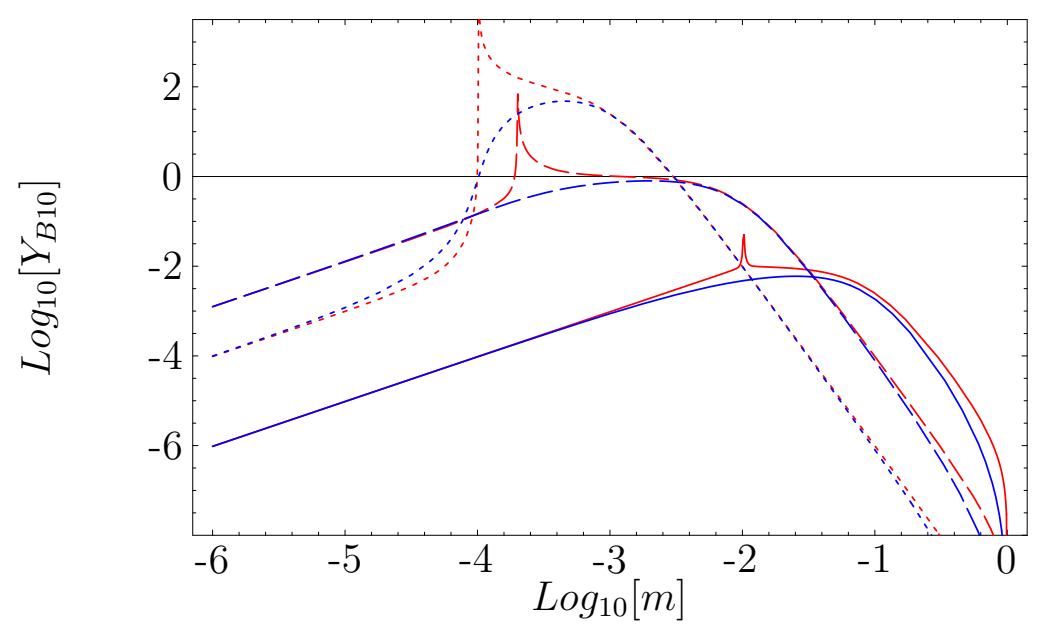

Figure 3: Log-Log plot of the asymmetry $Y_{B 10}$ (in arbitrary units) as a function of $m=m_{2} / m_{3}$ with the same conventions as in figure 2. For each curve there are 2 twins: the red spiky curve is the exact version of (4.9) computed for the phase maximising its value, while the smooth blue curve is the exact version of (4.7) for real positive parameters.

parameters, and that the relevant phase is given by

$$
A_{12}=e^{-i \delta_{R}}\left|A_{12}\right|
$$

The asymmetry (2.10) in the same approximation as (4.7) is then actually

$$
Y_{B 10} \propto \frac{\operatorname{Im}\left(A_{12}^{2}\right)}{A_{11}^{2}} \cdot \frac{M_{1}}{M_{2}} \cdot M_{1} \approx \sin \left(-2 \delta_{R}\right) \frac{|m| r^{2}|s|^{2}}{\left|m+r^{2}+s^{2}\right|^{5}}
$$

which has the same qualitative features as the previous expression $Y_{B 10}^{a p p r}$, with a further suppression by the factor $\left|\sin \left(-2 \delta_{R}\right)\right|<1$, and a possible enhancement when the denominator cancels, i.e. outside the range of validity of the approximations. Figure 3 shows that for a maximising value of the phase $\delta_{R}$, the correct asymmetry $Y_{B 10}$ is close to $Y_{B 10}^{a p p r}$ worked out without phases, after replacing $\operatorname{Im}\left(A_{12}^{2}\right)$ by $\left|A_{12}^{2}\right|=$ $A_{12}^{2}$. This approximation can be a useful guide for finding upper bounds in the full 3 flavours case, where the large number of phases makes it non-trivial.

To summarise, the lesson learned in this 2 flavours see-saw exercise is that

1. the right neutrinos mass ratio $M_{1} / M_{2}$ is maximised for a ratio of light neutrino masses $m=m_{1} / m_{2}$ which is either the squared ratio of Dirac masses $r^{2}=\left(m_{D 1} / m_{D 2}\right)^{2}$ or the squared sine of the light effective mixing angle $s^{2}=$ $\sin ^{2} \theta_{\text {eff }}$, whichever is largest,

2. the baryon asymmetry can be maximised for a similar or slightly larger value of $m$, 
3. Majorana phases cancellations can lead to further enhancements around that same value of $m$ (red curve on figure 2), but these can only be trusted after a careful resummation of this degenerate $M_{1} \approx M_{2}$ case;

4. for small mixing, the "inverted hierarchy" $m_{1} \gg m_{2}$ gives much smaller results than the "standard hierarchy" $m_{1} \ll m_{2}$.

Finally, we should also notice that this 2 flavours exercise was based on the approximate form (4.7) which, as (2.10), is only valid for $K>1$, and overestimates the asymmetry for lower $K$. For the results below we use the relation (2.9) which includes more realistic Boltzmann dilution.

\section{Results with 3 flavours}

Let us now discuss the more realistic situation of 3 light neutrino flavours. It turns out the baryon asymmetry is generically too small to be useful. Before concluding that $S O(10)$ see-saw leptogenesis is excluded, we must look for regions of parameters where the asymmetry can be maximised. As seen in the previous section, standard hierarchy is a better candidate in this respect. We will pass in review the various solutions to the solar neutrino deficit, starting by vacuum oscillations (VAC).

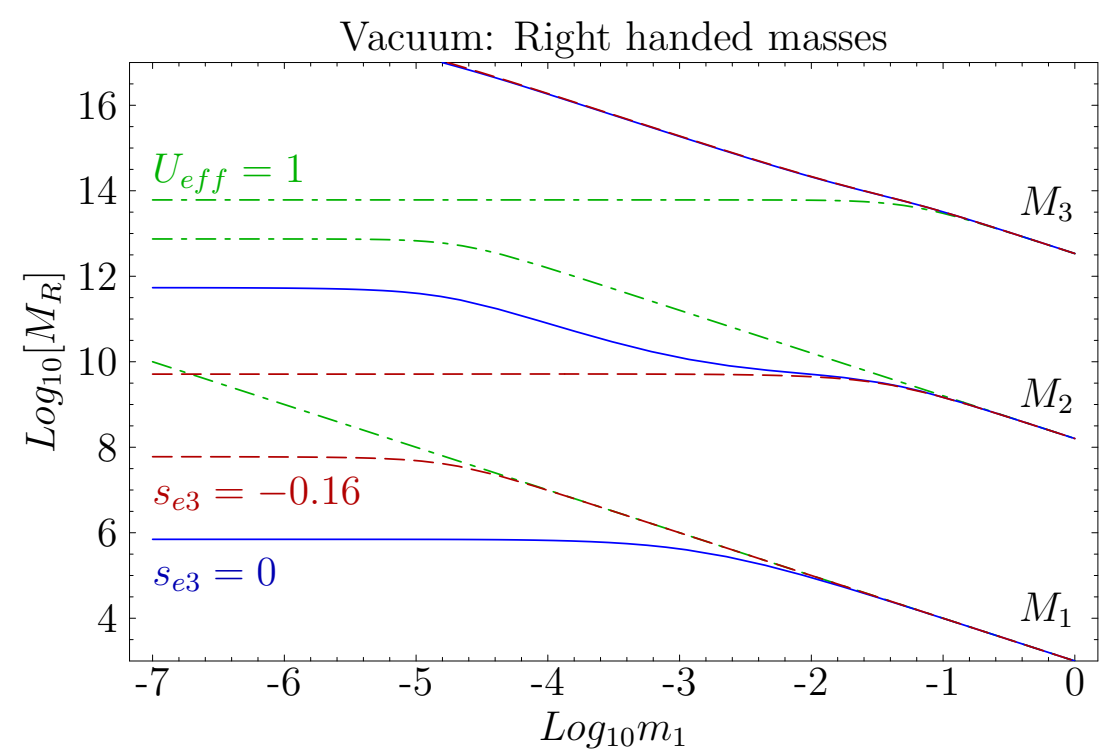

Figure 4: Masses of right-handed heavy Majorana neutrinos for vacuum oscillation of solar neutrinos $\left(\Delta m_{\text {sun }}=2.1510^{-5} \mathrm{eV}\right)$. The dash-dotted curves completely ignore mixing $\left(U_{\text {eff }}=1\right)$, the plain curves are for real CKM mixing, maximal atmospheric and solar mixing but vanishing $s_{e 3}$ while the dashed curves tune $s_{e 3}$ to maximise $M_{1} / M_{2}$.

In view of the previous section, a good starting point to maximise the baryon asymmetry is to try and make the lightest right-handed masses $M_{1}$ and $M_{2}$ as close as possible to each other. If there were no mixing, $U_{\text {eff }}$ in (3.14) would be diagonal 
and right-handed masses would follow the dash-dotted curves of figure 1 . For large $m_{1}$ (degenerate limit) they draw 3 parallel lines separated by large Dirac mass hierarchies. With decreasing $m_{1}$, all $M_{R}$ 's grow until $m_{1}=\Delta m_{\text {atm }} \approx 10^{-1} \mathrm{eV}$, below which $M_{3}$ levels off at $m_{t}^{2} / 9 \Delta m_{a t m} \approx 10^{14} \mathrm{GeV}$. Meanwhile $M_{1,2}$ continue growing together until $m_{1}=\Delta m_{\text {sun }} \approx 10^{-5} \mathrm{eV}$, where $M_{2}$ stops at about the same value. Further decreasing $m_{1}$ then only affects $M_{1}$, which becomes degenerate with $M_{2}$ for extremely low $m_{1} \approx 10^{-11} \mathrm{eV}$.

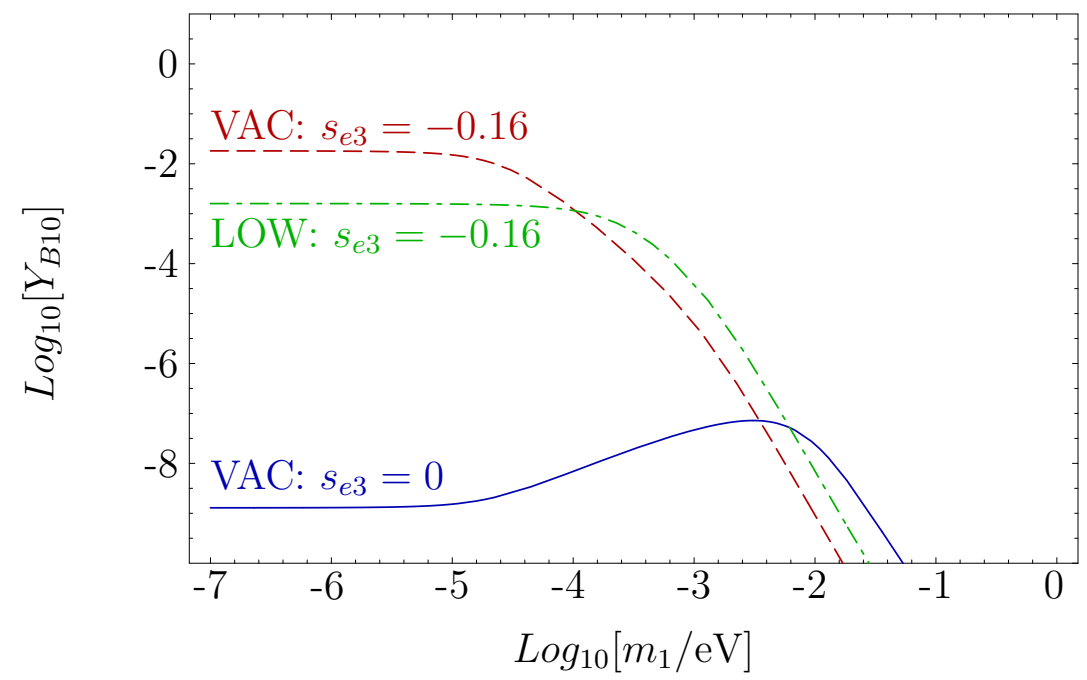

Figure 5: The baryon asymmetry $Y_{B 10}$ for LOW and VAC oscillations of solar neutrinos. The plain curve is the vacuum case for vanishing $s_{e 3}$. The gain from tuning the value $s_{e 3}=-0.16$ is shown for the VAC (dashed) and LOW (dash-dotted) cases, taking for each case the maximising value of the phase $\delta_{13}$.

If we now turn on the largest allowed atmospheric and solar mixing, we get the plain curves in figure 4 . Decreasing $m_{1}$ from the unaffected degenerate limit, $M_{3}$ starts again levelling off at $m_{1}=\Delta m_{a t m}$. But the maximal atmospheric mixing immediately induces the type of "level crossing" seen in the previous section, which effectively exchanges $M_{2}$ and $M_{3} . M_{2}$ thus levels off at a much lower value $\approx 10^{10} \mathrm{GeV}$ which offers a better possibility for $M_{1}$ to catch up. However, despite keeping $s_{e 3}=0$, CKM mixing in eq. (3.16) still induces a non-trivial $\left|s_{13 e f f}\right| \approx 0.16$ which stops the growth of $M_{1}$ below $m_{1}=\left|s_{13 e f f}\right|^{2} \Delta m_{\text {atm }} \approx 10^{-3} \mathrm{eV} . \quad M_{2}$ on the contrary starts growing again until it is hit by the solar mixing at $m_{1} \approx \Delta m_{\text {sun }}$. We thus expect a maximum baryon asymmetry around $m_{1} \approx 10^{-3} \mathrm{eV}$ in this case. A plot of the full 3 flavour asymmetry (plain curve in figure 5) confirms this expectation, and further shows that this maximum is at least 5 orders of magnitude too low. The shape of the asymmetry nicely fits the picture derived in the previous section, once we recall that for $m_{1}<\Delta m_{\text {sun }}$ both $M_{1}$ and $M_{2}$ stay constant. It is also worth noticing that at this stage, the only source of $\mathrm{CP}$ violation is $\delta^{C K M}$, and that it drives the right 
sign for the baryon asymmetry, albeit with a small amplitude.
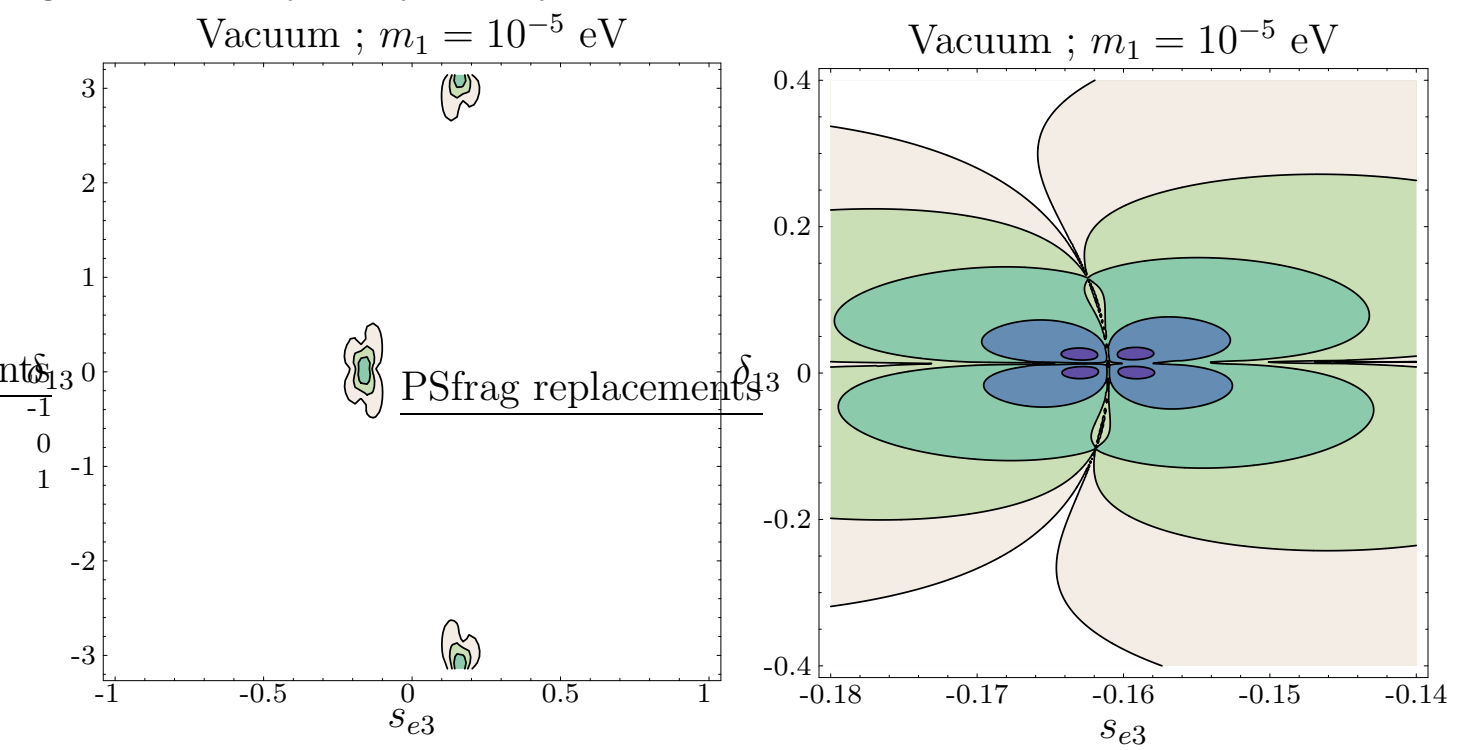

Figure 6: Sensitivity of the baryon asymmetry on the matrix element $U_{e 3}=s_{e 3} e^{i \delta_{13}}$ most constrained by CHOOZ. The levels of $Y_{B 10}$ shown are $10^{-6}, 10^{-5} \ldots 10^{-2}$, the latter being only obtained on the darkest dots around $\left(s_{e 3}=-0.16, \delta_{13}=0.0\right)$ at the centre of the zoom on the right.

To get larger results, we may use the freedom left by the CHOOZ experiment

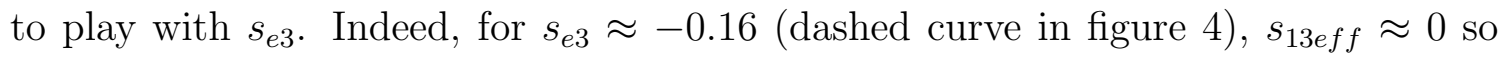
that $M_{1}$ is decoupled from the value of $m_{3}$, and continues rising up to $m_{u}^{2} / 9 \Delta m_{\text {sun }} \approx$ $10^{8} \mathrm{GeV}$. This may give a rise in the asymmetry down to $m_{1} \approx 10^{-5} \mathrm{eV}$. Notice that for such a value, $K \approx 1$ which minimises dilution effects, $M_{1} \approx 10^{8} \mathrm{GeV}$ which is safe w.r.t. gravitino bounds on inflation in SUSY versions of this scenario, and $M_{3} \approx 10^{16} \mathrm{GeV}$ which is marginally compatible with the GUT scale. It is worth detailing where the special value $s_{e 3} \approx-0.22 s_{\text {atm }}$ comes from. In the expression of $U_{\text {eff }}$, we may in a first approximation neglect all CKM mixing except the Cabbibo $V_{12}^{C K M}$. Then keeping terms at most linear in $s_{12}^{C K M}$ and $s_{e 3}$, we may write:

$$
\begin{aligned}
U_{e f f} & \approx V_{12}^{C K M} U_{23} U_{13} U_{12} \\
& =U_{23} U_{13}\left[U_{13}^{\dagger} U_{23}^{\dagger} V_{12}^{C K M} U_{23} U_{13}\right] U_{12} \\
& \approx U_{23} U_{13} V_{13}^{\prime} V_{12}^{\prime} U_{12} \\
& \doteq U_{23 e f f} U_{13 e f f} U_{12 e f f}
\end{aligned}
$$

with the commutator $V^{\prime}$ matrices parameterised like (3.17) with $s_{12}^{\prime} \approx c_{a t m} s_{12}^{C K M}$ and $s_{13}^{\prime} \approx s_{a t m} e^{i \delta_{23}} s_{12}^{C K M}$. Cancelling $s_{13 e f f}$ then requires to satisfy the complex equation

$$
U_{e 3}=s_{e 3} e^{-i \delta_{13}} \approx-s_{a t m} e^{-i \delta_{23}} s_{12}^{C K M}=-0.16 e^{-i \delta_{23}}
$$

which is illustrated for $\delta_{23}=0$ in figure 6. One sees the extreme tuning on both the modulus and the phase of $U_{e 3}$, needed to achieve an enhancement in the four dark 
central dots of the zoom. Even the sign of the asymmetry violently flips in the four quadrants of this zoom.

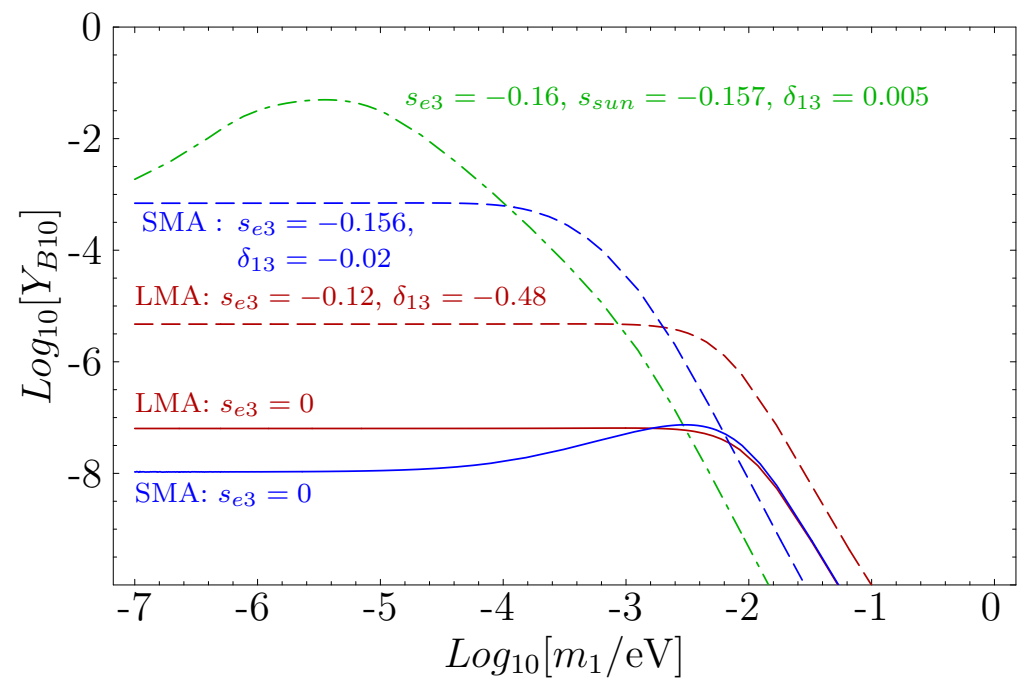

Figure 7: The baryon asymmetry $Y_{B 10}$ for MSW oscillations of solar neutrinos with various mixing angles. The plain curves are for vanishing $s_{e 3}$ with no phase except $\delta^{C K M}$. The dashed SMA (resp. LMA) curves are for $\delta_{13}=-0.02$ (resp. -0.1) . Only the particular dash-dotted curve $\left(s_{e 3}=-0.16, s_{\text {sun }}=-0.156, \delta_{13}=0.005\right)$ can nearly produce enough asymmetry, but it can not account for the solar neutrino deficit.

The previous discussion can be repeated for the LOW solution, but the larger value of $\Delta m_{\text {sun }}^{2}$ leaves less room for the asymmetry to grow before levelling off. As seen in figure 5, the maximum that can hoped for is 2 orders of magnitude too low.

If we now turn to MSW solutions of the solar neutrino deficit, we get a still larger value of $\Delta m_{\text {sun }}$, and there is too little room for $M_{1}$ to grow between $m_{1} \approx \Delta m_{\text {atm }}$ and $m_{1} \approx \Delta m_{\text {sun }}$. However, it can grow for smaller $m_{1}$, provided both $s_{13 e f f}=s_{12 e f f}=0$. This is in principle possible, but now requires very special values for both $s_{e 3} \approx-0.16$ (as previously) and $s_{\text {sun }} \approx-s_{12}^{C K M} c_{\text {atm }} \approx-0.16$ (see eq. 5.3). This last value of $s_{\text {sun }}$ is incompatible with any possible MSW solution, unless taking $s_{12}^{C K M}$ away from its quark-lepton symmetry value, which is illustrated as a function of $m_{1}$ and $s_{\text {sun }}$ on figures 7 and 8 .

With a standard mass hierarchy, we thus see that vacuum oscillations are the closest to account both for the solar neutrinos deficit and the baryon asymmetry of the universe. With the same line of reasoning, it is easy to see that with inverted hierarchy $\left(m_{3} \ll m_{1} \ll m_{2}\right)$ the asymmetry is even smaller, because of a larger $M_{1}-$ $M_{2}$ gap coming from the bound $M_{1}<m_{u}^{2} / 6 \Delta m_{\text {atm }}$. Indeed, as the free parameter $m_{3}$ is lowered below $\Delta m_{\text {atm }}$, two $M_{R}$ eigenvalues must now stop growing instead of one. There are then two possibilities: if $M_{1}$ is the only one that grows in the absence of mixing, then maximal atmospheric mixing stops its growth once $m_{3}<s_{\text {atm }} \Delta m_{a t m}$. 


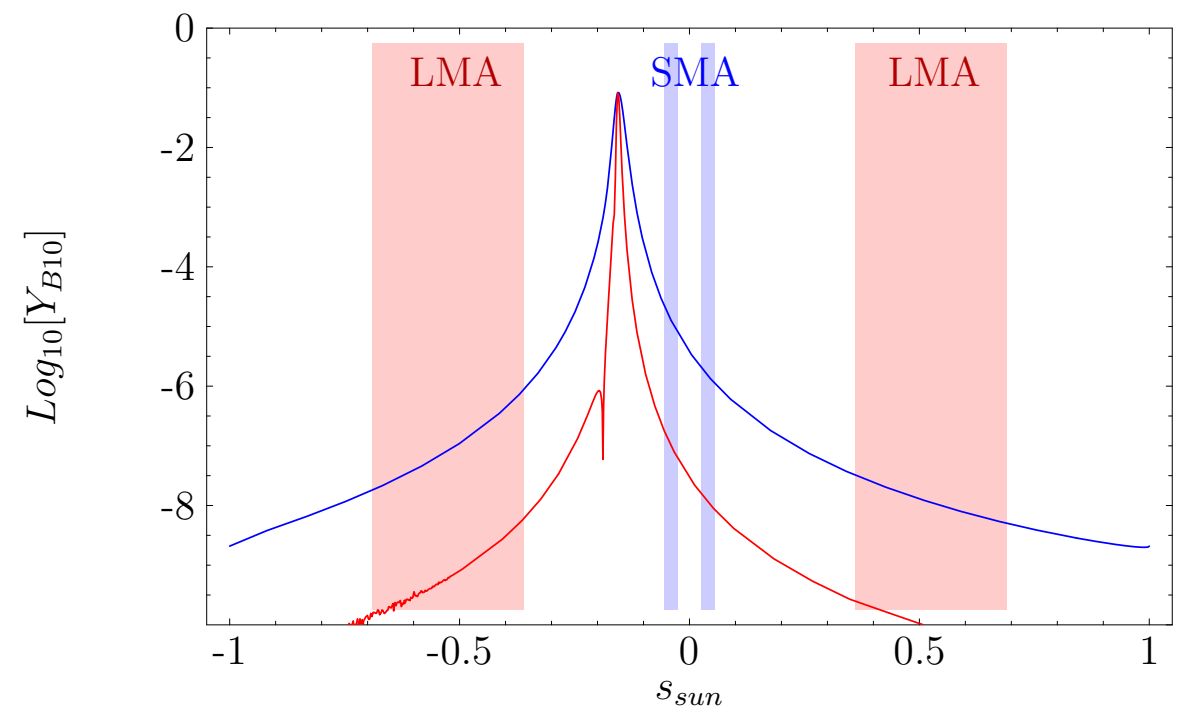

Figure 8: The baryon asymmetry $Y_{B 10}$ for MSW oscillations of solar neutrinos (LMA: lower red, SMA: upper blue), as a function of $s_{\text {sun }}$. The maximum is nearly large enough (and sharply peaked) for $m_{1} \approx 10^{-5}$ if $s_{e 3}=-0.16$, but lies just between the small and large mixing angle rectangles.

If on the other hand $M_{1}$ stops growing without mixing, then mixing won't help, as it can only induce "levels repulsion", not attraction. In both cases, $M_{1}$ is at most $10^{4} \mathrm{GeV}$, and $M_{1} / M_{2}$ cannot exceed $m_{u}^{2} / m_{c}^{2} \approx 10^{-6}$ which makes the asymmetry too small.

Let us now review the effect of the various phases for trying to reach a sufficient asymmetry. In the discussion above, we have only switched on the minimal phases necessary to get a non-trivial result, namely $\delta^{C K M}$ which is unavoidable in our quarklepton symmetry set-up, and $\delta_{13}$ which was necessary to enforce the cancellation (5.5). From this equation, it is clear that if $\delta_{23}$ were non zero, it would merely shift the optimal value of $\delta_{13}$, the asymmetry being a function of the combination $\delta_{13}-\delta_{23}$ in the region of the low $m_{1}$ peak. An non-zero $\delta_{12}$ would induce a much smaller shift, through the sub-leading corrections to (5.5). Less trivial are the effects of the Majorana phases $\phi_{1,2}$. As suggested by equation (4.9), these can lead to cancellations and near degenerate right-handed masses for a fine tuned value of one of the phases and of $m_{1}$. However, the peak in the decay asymmetries $\epsilon_{1,2}$ and $\epsilon_{1,3}$ tend to cancel each other, and get further reduced by a similar peak in $K_{1}$, so that no enhancement of the final asymmetry can be produced for the large $m_{1} \approx 10^{-3} \mathrm{eV}$ where it could occur. 


\section{Conclusions and perspectives}

In this work, we have studied the possible relations between neutrino oscillations and the baryon asymmetry of the universe produced by equilibrium decays of right handed neutrinos, assuming an $S O(10)$ see-saw mechanism for neutrino masses. As explained, this is a minimal predictive set of assumptions to study such relations. We find that the produced asymmetry is generically six orders of magnitude too small

because of the huge Dirac masses hierarchy assumed $\left(r^{2}=m_{u}^{2} / m_{c}^{2} \approx 10^{-6}\right)$. The closest one can come to the observed asymmetry in this framework is at least one order of magnitude too small and requires vacuum oscillations of solar neutrinos, with standard hierarchy and very specific values for the least constrained neutrino parameters: 1$)$ the lightest mass $\left(m_{1} \approx \Delta m_{\text {sun }} \approx 10^{-5} \mathrm{eV}\right)$, and 2$)$ the heavy component of the electron neutrino $\left(\left|s_{e 3}\right|=\left|s_{\text {atm }}\right| \cdot\left|\sin \left(\theta_{\text {Cabbibo }}\right)\right| \approx 0.16\right)$.

This result calls for several extensions and refinements. It would first of all be more satisfactory to get the right-handed mass relations we tend to need out of some theoretical mass model for all particles. This seems quite challenging at the moment. Nice family symmetry models like [44, 45] for instance, generically have a much larger $M_{1} / M_{2}$ hierarchy than we found necessary for leptogenesis.

Second, one may question our crude implementation of the $S O(10)$ relations between quarks and leptons Dirac-masses. We simply used $m_{\text {lepton }}=m_{\text {quark }} / 3$, which fails by about a factor 3 for the muon-strange mass ratio. Since the asymmetry goes like $r^{2}$ (see eq. 4.7), such small factors should not qualitatively change our conclusions; taking 10 instead of $1 \mathrm{MeV}$ for the lowest neutrino Dirac mass could for instance barely bring the vacuum oscillation solution up to the required asymmetry $Y_{B 10} \approx 1$. The correct running of neutrino Yukawa couplings may have important effects on the mixing, which could change the interesting value of $s_{e 3}$, and for instance make it inaccessible to first generation long baseline experiments, or on the contrary, already excluded by $\mathrm{CHOOZ}$.

Our conclusions seemingly contradict previous literature on the subject, which usually left the impression that leptogenesis could work without restrictions. This comes from our maybe over-restrictive framework, insisting on both $S O(10)$ relations, and recent neutrino oscillations data. Let us review some of these discrepancies. In reference [46, 24], the authors use $S O(10)$-inspired see-saw relations like eq. 3.14 and conclude that leptogenesis is generically possible. However, the values taken in the final analysis are hard to reconcile with maximal atmospheric mixing, which at the time was not as firmly established as today. In subsequent works [47, 48], maximal atmospheric mixing is well taken into account, but the Dirac mass pattern considered is derived from a family symmetry in an $S U(5)$ framework; this gives typically larger "CKM" mixing and much weaker Dirac hierarchy than the simple quark-lepton ansatz we considered, both of which increase the asymmetry. In reference [49], it is shown that leptogenesis can be reconciled with SMA solar neutrinos. 
But the Dirac masses needed badly violate $S O(10)$ relations. Finally, another way to loosen the strong $S O(10)$ constraints is to invoke what could be coined "see-saw in the scalar sector", namely a small v.e.v. for a scalar triplet that directly contributes to the left neutrino Majorana mass [31]. This however introduces a new parameter, and non-trivial constraints on the scalar sector as in [50].

Note added: While this work was being revised, others [51, [52] have confirmed the important effect of $U_{e 3}$ in the framework discussed here. It should however be stressed that the type of random scan used in these works requires a very fine graining to hit the maximal possible value: at least $10^{5}$ points in each $U_{e 3}$ plane, as seen figure 6. After the recent KamLAND results[41], the only surviving LMA solution requires an optimal value of $\left|U_{e 3}\right| \sim 0.12$ which is but slightly lower than the VAC case detailed in this work. However, reconciling thermal leptogenesis with an $S O(10)$ mass pattern seems even harder than before.

\section{References}

[1] G. Steigman, The baryon density through the (cosmological) ages, Fortsch. Phys. 50 (2002) 562-568, http://arXiv.org/abs/astro-ph/0202187.

[2] A. D. Dolgov, Nongut baryogenesis, Phys. Rept. 222 (1992) 309-386.

[3] M. E. Shaposhnikov, Baryon asymmetry of the universe in standard electroweak theory, Nucl. Phys. B287 (1987) 757.

[4] V. A. Rubakov and M. E. Shaposhnikov, Electroweak baryon number non-conservation in the early universe and in high-energy collisions, Usp. Fiz. Nauk 166 (1996) 493-537, [hep-ph/9603208].

[5] A. D. Sakharov, Violation of cp invariance, c asymmetry, and baryon asymmetry of the universe, Pisma Zh. Eksp. Teor. Fiz. 5 (1967) 32-35.

[6] M. B. Gavela, P. Hernandez, J. Orloff, and O. Pene, Standard model cp violation and baryon asymmetry, Mod. Phys. Lett. A9 (1994) 795-810, hep-ph/9312215.

[7] M. B. Gavela, P. Hernandez, J. Orloff, O. Pene, and C. Quimbay, Standard model cp violation and baryon asymmetry. part 2: Finite temperature, Nucl. Phys. B430 (1994) 382-426, hep-ph/9406289.

[8] A. I. Bochkarev, S. V. Kuzmin, and M. E. Shaposhnikov, Electroweak baryogenesis and the higgs boson mass problem, Phys. Lett. B244 (1990) 275. 
[9] M. Carena, M. Quiros, and C. E. M. Wagner, Electroweak baryogenesis and higgs and stop searches at lep and the tevatron, Nucl. Phys. B524 (1998) 3, hep-ph/9710401.

[10] V. A. Kuzmin, V. A. Rubakov, and M. E. Shaposhnikov, On the anomalous electroweak baryon number nonconservation in the early universe, Phys. Lett. B155 (1985) 36.

[11] S. T. Petcov, Neutrino mixing and oscillations in 1999 and beyond, in Cape Town 1999, Weak Interactions and Neutrinos, pp. 305-319, 1999. hep-ph/9907216.

[12] T. Yanagida, Workshop on unified theories, 1979. KEK report 79-18.

[13] M. Gell-Mann, P. Ramond, and R. Slansky, Complex spinors and unified theories, in Supergravity (P. van Nieuwenhuizen and D. Freedman, eds.), North-Holland, 1979. Print-80-0576 (CERN).

[14] T. Yanagida, Horizontal symmetry and masses of neutrinos, Prog. Theor. Phys. 64 (1980) 1103.

[15] R. N. Mohapatra and G. Senjanovic, Neutrino mass and spontaneous parity violation, Phys. Rev. Lett. 44 (1980) 912.

[16] M. Fukugita and T. Yanagida, Baryogenesis without grand unification, Phys. Lett. B174 (1986) 45.

[17] J. A. Harvey and M. S. Turner, Cosmological baryon and lepton number in the presence of electroweak fermion number violation, Phys. Rev. D42 (1990) 3344-3349.

[18] A. Pilaftsis, Heavy majorana neutrinos and baryogenesis, Int. J. Mod. Phys. A14 (1999) 1811, hep-ph/9812256.

[19] M. Plumacher, Baryon asymmetry, lepton mixing and so(10) unification, in Tegernsee 1998, New Trends in Neutrino Physics, pp. 299-308, 1998. http://arXiv.org/abs/hep-ph/9809265.

[20] W. Buchmuller and M. Plumacher, Cp asymmetry in majorana neutrino decays, Phys. Lett. B431 (1998) 354-362, http://arXiv.org/abs/hep-ph/9710460.

[21] L. Covi, E. Roulet, and F. Vissani, Cp violating decays in leptogenesis scenarios, Phys. Lett. B384 (1996) 169-174, hep-ph/9605319.

[22] J. M. Frere, F. S. Ling, M. H. G. Tytgat, and V. V. Elewyck, Leptogenesis with virtual majorana neutrinos, Phys. Rev. D60 (1999) 016005, hep-ph/9901337. 
[23] E. W. Kolb and M. Turner, The Early Universe, ch. 6. Frontiers in Physics. Addison-Wesley, 1990.

[24] M. Plumacher, Baryon asymmetry, neutrino mixing and supersymmetric so(10) unification, Nucl. Phys. B530 (1998) 207, hep-ph/9704231.

[25] W. Buchmuller and M. Plumacher, Neutrino masses and the baryon asymmetry, Int. J. Mod. Phys. A15 (2000) 5047-5086, http://arXiv.org/abs/hep-ph/0007176.

[26] W. Buchmuller, P. Di Bari, and M. Plumacher, Cosmic microwave background, matter-antimatter asymmetry and neutrino masses, Nucl. Phys. B643 (2002) 367-390, [hep-ph/0205349].

[27] H. B. Nielsen and Y. Takanishi, Baryogenesis via lepton number violation in anti-gut model, Phys. Lett. B507 (2001) 241-251, http://arXiv.org/abs/hep-ph/0101307.

[28] M. Hirsch and S. F. King, Leptogenesis with single right-handed neutrino dominance, Phys. Rev. D64 (2001) 113005, http://arXiv.org/abs/hep-ph/0107014.

[29] R. Barbieri, P. Creminelli, A. Strumia, and N. Tetradis, Baryogenesis through leptogenesis, Nucl. Phys. B575 (2000) 61-77, http://arXiv.org/abs/hep-ph/9911315.

[30] H. Fritzsch and P. Minkowski, Unified interactions of leptons and hadrons, Ann. Phys. 93 (1975) 193-266.

[31] P. B. P. Rabindra N. Mohapatra, Massive Neutrinos in Physics and Astrophysics, vol. 60 of Lecture Notes in Physics. World Scientific, 1998.

[32] Particle Data Group Collaboration, K. Hagiwara et. al., Review of particle physics, Phys. Rev. D66 (2002) 010001.

[33] S. M. Bilenkii, C. Giunti, and W. Grimus, Phenomenology of neutrino oscillations, Prog. Part. Nucl. Phys. 43 (1999) 1, hep-ph/9812360].

[34] L. Baudis et. al., Limits on the majorana neutrino mass in the 0.1-ev range, Phys. Rev. Lett. 83 (1999) 41, hep-ex/9902014.

[35] C. Giunti, Neutrinoless double-beta decay with three or four neutrino mixing, Phys. Rev. D61 (2000) 036002, hep-ph/9906275.

[36] F. Vissani, Signal of neutrinoless double beta decay, neutrino spectrum and oscillation scenarios, JHEP 06 (1999) 022, hep-ph/9906525.

[37] T. Endoh, T. Morozumi, T. Onogi, and A. Purwanto, $C p$ violation in seesaw model, Phys. Rev. D64 (2001) 013006, http://arXiv.org/abs/hep-ph/0012345. 
[38] M. Apollonio et. al., Limits on neutrino oscillations from the chooz experiment, Phys. Lett. B466 (1999) 415, [hep-ex/9907037].

[39] M. C. Gonzalez-Garcia and Y. Nir, Developments in neutrino physics, http://arXiv.org/abs/hep-ph/0202058.

[40] SNO Collaboration, Q. R. Ahmad et. al., Measurement of day and night neutrino energy spectra at sno and constraints on neutrino mixing parameters, Phys. Rev. Lett. 89 (2002) 011302, http://arXiv.org/abs/nucl-ex/0204009.

[41] KamLAND Collaboration, K. Eguchi et. al., First results from kamland: Evidence for reactor anti- neutrino disappearance, Phys. Rev. Lett. 90 (2003) 021802, hep-ex/0212021.

[42] T. Yanagida and M. Yoshimura, Neutrino mixing in a class of grand unified theories, Phys. Lett. B97 (1980) 99.

[43] E. K. Akhmedov, Small entries of neutrino mass matrices, Phys. Lett. B467 (1999) 95, hep-ph/9909217.

[44] N. Irges, S. Lavignac, and P. Ramond, Predictions from an anomalous u(1) model of yukawa hierarchies, Phys. Rev. D58 (1998) 035003, [hep-ph/9802334].

[45] J. Sato and T. Yanagida, Large lepton mixing in a coset-space family unification on e(7)/su(5) x u(1)**3, Phys. Lett. B430 (1998) 127-131, hep-ph/9710516.

[46] W. Buchmuller and M. Plumacher, Baryon asymmetry and neutrino mixing, Phys. Lett. B389 (1996) 73-77, hhttp://arXiv.org/abs/hep-ph/9608308].

[47] W. Buchmuller and T. Yanagida, Quark lepton mass hierarchies and the baryon asymmetry, Phys. Lett. B445 (1999) 399-402, [hep-ph/9810308].

[48] W. Buchmuller and M. Plumacher, Matter antimatter asymmetry and neutrino properties, Phys. Rept. 320 (1999) 329-339, http://arXiv.org/abs/hep-ph/9904310.

[49] H. Goldberg, Leptogenesis and the small-angle msw solution, Phys. Lett. B474 (2000) 389, hep-ph/9909477.

[50] A. S. Joshipura and E. A. Paschos, Constraining leptogenesis from laboratory experiments, hep-ph/9906498.

[51] D. Falcone and F. Tramontano, Leptogenesis and neutrino parameters, Phys. Rev. D63 (2001) 073007, hep-ph/0011053.

[52] G. C. Branco, R. Gonzalez Felipe, F. R. Joaquim, and M. N. Rebelo, Leptogenesis, cp violation and neutrino data: What can we learn?, Nucl. Phys. B640 (2002) 202-232, [http://arXiv.org/abs/hep-ph/0202030]. 